\section{Spatially-Linked Manufacturing Data to Support Data Analysis}

Gl_Forum 2017, Issue 1

Page: 126 - 140

Full Paper

Corresponding Author:

stefan.schabus@infineon.com

DOI: 10.1553/giscience2017_01_s126

\author{
Stefan Schabus' and Johannes Scholz² \\ IInfineon Technologies Austria AG, Villach, Austria \\ ${ }^{2} \mathrm{Graz}$ University of Technology, Institute of Geodesy, Graz, Austria
}

\begin{abstract}
The paper presents a Linked Data approach within a manufacturing organization to foster sharing, reusing, integrating and the collaborative analysis of datasets originating from different business units and heterogeneous data sources. The paper relies on a semiconductor company that serves as case study. The authors elaborate on manufacturing data and their representation in a spatially-enabled graph database, and as Linked Data based on an ontology describing the indoor space and production processes. A graph database enables data sharing as well as the semantic search and retrieval of data utilizing web-based services. The results present the analysis of historic, future and spatio-temporal data as well as the analysis of similarities of semanticallyannotated linked manufacturing data.
\end{abstract}

\title{
Keywords:
}

linked manufacturing data, semantic annotations, smart manufacturing, graph databases

\section{Introduction}

In manufacturing industries, huge amounts of data with an inherent spatio-temporal dimension are generated during manufacturing processes. These manufacturing data satisfy the criteria for Big Data (De Mauro et al., 2016, p. 128): 'Big Data is the Information asset characterized by such a High Volume, Velocity and Variety to require specific Technology and Analytical Methods for its transformation into Value.' Manufacturing data comprise high volume and high velocity based on tracking of positions, processes and quality. The question of variety is tackled in this research, as the approach aims to solve semantic interoperability. Manufacturing data are necessary to support decision-making in near real-time. They are a result of measurement values provided by production equipment and sensors present in the production line. By monitoring the position of production assets using an indoor positioning system, spatial data are created. Combining them with manufacturing data, they become strongly intertwined with the spatio-temporal dimension (Davis et al., 2012; Zuehlke, 2010). Hence, it is important to generate knowledge from this data to support decision-making and learning from historical events. To do so, the data, which are created by different systems, 
need to be connected and integrated. This raises issues of semantic interoperability: there is a lack of explicit semantics, because there exist hardly any semantically-annotated base data from manufacturing equipment.

In order to overcome interoperability issues, the authors propose to semantically annotate the data, by making use of relevant ontologies in the field (Scholz \& Schabus, 2014; Schabus \& Scholz, 2015). This opens up possibilities for data- and knowledge-sharing with regard to existing spatio-temporal data (Klien, 2007), as has been shown for the case of Spatial Data Infrastructures (Lutz et al., 2009). The Linked Data paradigm is a recent shift in Geographic Information Science, offering a new way of structuring, publishing, discovering, accessing and integrating data (Kuhn et al., 2014). Linked Data are a collection of design principles and technologies around the Web of Data or Semantic Web. There, data are represented as triples (subject, predicate and object) in the Resource Description Framework (RDF) format. Data formatted in this way should conform to the Linked Data principles, which ensure that data are machine-readable, have a uniform resource identifier (URI) to denote things, and use the World Wide Web Consortium (W3C) standards, such as Web Ontology Language (OWL) or RDF. Data linking out to other data should use URIs to create an interconnected graph of knowledge. As RDF data are machine-readable, they seem well suited for information-sharing and for being shared between different applications.

The application context is a specific indoor space - a semiconductor manufacturing company. The indoor environment under review looks different from buildings for office or residential use, as no rooms are present and there are specific cleanroom restrictions. The production processes carried out in the production line have highly flexible characteristics, which means that the manufacturing line is not of 'conveyor belt' type with a fixed processing chain. This is due to the fact that several hundred production steps are necessary to create the finished product. Additionally, most production steps can be carried out on several pieces of equipment, which are often geographically dispersed over the production facility.

The research question of this paper can be summarized as: 'Does the translation of relational manufacturing data into semantically-annotated linked data contribute to new knowledgegeneration based on data links and data analysis, i.e. the identification of similarities?' The authors focus on Linked Data in the context of manufacturing data as a new possibility to overcome the interoperability issues of heterogeneous data sources in manufacturing. Linked manufacturing data allow the sharing of semantically-annotated data across different business divisions of an organization. This increases the possibility of developing a decision support system for a manufacturer as well as leveraging analytical 'learning' from historical data.

The structure of the paper is as follows: section 2 covers relevant work; manufacturing data and their representation as Linked Manufacturing Data are the subject of section 3; section 4 presents the visualization of linked data and the analysis of similarities based on data semantics. Finally, we present a critical analysis of the results. 


\section{Relevant Work}

This section covers related work that contributes to fields of scientific expertise covered in the paper. The authors emphasize indoor geography, modelling indoor space, and indoor navigation. In addition, the literature covers ontologies, semantics and linked data with respect to manufacturing data.

The average person spends $90 \%$ of their time inside buildings (Klepeis et al., 2001). Consequently, a number of research activities are currently being carried out to apply Geoinformation indoors. Early research elaborates on indoor modelling and pathfinding. In Raubal \& Worboys (1999), for example, an airport serves as an example for agent-based indoor wayfinding. Several approaches to model indoor spaces exist, ranging from reducing the indoor space to a graph (e.g. Goetz \& Zipf, 2011) to Building Information Models (e.g., Howell \& Batcheler, 2005). Yang \& Worboys (2015) as well as Scholz \& Schabus (2014) employed ontologies to model indoor space. Scholz \& Schabus (2014) used ontologies to model an indoor production environment with several requirements. Additionally, Schabus \& Scholz (2015) proposed that space and time can help to improve decision processes in production environments.

Smart manufacturing is one of the main research fields to support decision-making in indoor production environm6ents, and to facilitate competitiveness (Davis et al., 2012). Most manufacturers collect data from processes, with explicit and implicit spatio-temporal reference. There is therefore a need to analyse and visualize such data. Data visualization in a spatio-temporal manner, using geovisual analytics, enables humans to identify patterns (Compieta et al., 2007; Andrienko et al., 2007; von Landesberger et al., 2016). Near real-time visualization could be of potential interest thanks to the emergence of wearable devices for employees and managers (Osswald et al., 2013). These devices allow a virtual view of performance and states of the manufacturing environment. Furthermore, spatio-temporal patterns can help to develop strategies and technologies to increase manufacturing efficiency - i.e. cost savings and increased performance (Nyström et al., 2006).

Semantics and ontologies are used in spatio-temporal modelling and have been discussed in scientific literature since the 1980s (Smith, 2001). Gruber (1993) defines an ontology as the formal specification of a shared conceptualization. A so-called domain ontology describes the specific domain in a general way, resulting in a formal description of the content and behaviour of a part of the physical world (Raubal \& Worboys, 1999). Davis (1990) describes the elements of an ontology as entities, relations and applied rules. Grenon \& Smith (2004) describe dynamic spatial ontologies that are capable of representing spatial relations. Types of ontologies are defined by Sowa (2014), who focuses on two in particular: a single large ontology or a collection of microworlds. These are described in greater detail by Yang \& Worboys (2011). Sowa (2014) defines an upper-ontology as the most generic way to describe a concept at a basic level. The upper-ontology subsumes the domain ontology and the task ontology, which describe either the environment or the task - for example, navigation. The most specific ontology is the application ontology, combining the task and the domain in one single large ontology (Sowa, 2014). 


\section{Manufacturing Data and their Representation}

To pave the way for the representation of manufacturing data as Linked Data (LD), there are a number of prerequisites that have to be fulfilled to transfer raw data into RDF. This section elaborates on a semantic description of manufacturing data, the indoor space and navigation actions, by utilizing ontologies. The authors use graph databases as physical storage for the generic ontology, as well as for the manufacturing data. To make manufacturing data ready for publishing as LD and using them thereafter, several issues need to be resolved. First, an appropriate semantic definition of the Universe of Discourse is necessary. The general concept of the Universe of Discourse is described by Boole (1854, p. 42):

'In every discourse, whether of the mind conversing with its own thoughts, or of the individual in his intercourse with others, there is an assumed or expressed limit within which the subjects of its operation are confined. [...] Now, whatever may be the extent of the field within which all the objects of our discourse are found, that field may properly be termed the universe of discourse.'

In this paper, we restrict ourselves to a semiconductor manufacturing company, focusing on the manufacturing processes taking place in cleanroom facilities. In order to model the special indoor space, we utilize a navigation and indoor space ontology, describing the indoor space and the production processes at hand (Scholz \& Schabus, 2014; Schabus \& Scholz, 2015), referred to as IndoorOntology::Production in the remainder of this article.

For the IndoorOntology::Production, Scholz \& Schabus (2014) developed a two-folded ontology in order to represent the indoor space and the manufacturing processes:

- Task ontology: indoor navigation ontology

- Domain ontology: production environment.

The main elements of the indoor space covered by the domain ontology are:

- ProductionUnit: describes the equipment pieces necessary for carrying out manufacturing processes; it is also used for facilities to store or deposit production assets;

- Corridor: denotes the spaces in a cleanroom that are walkable by humans and traversable by production assets;

- Barrier: limits the movement behaviour in the production line;

- Restriction: denotes specific restrictions that are due to cleanroom quality, contamination risks or maintenance, or to production data;

- Accessnode: links indoor and outdoor space (Schabus et al., 2015)

- NavigationAgent: the production asset.

The elements of the task ontology are navigation tasks and events (e.g. turn left or right), a graph-based structure with nodes and edges that enable routing in the indoor space. Additionally, the ontology includes the indoor space and affordances of production assets (i.e. the NavigationAgents). An example of such an affordance is that a staircase does not 
allow traversing a box of a specific product type, whereas an elevator does, which is due to the risk of damaging the production assets.

\section{Linked Data concept for Manufacturing Data}

In this section, we aim to show how heterogeneous manufacturing data, created by a number of heterogeneous systems and sensors, can be represented as LD. This section highlights the general concept and relations between IndoorOntology::Production, manufacturing and spatial data. In addition, the raw datasets are briefly described as a basis for understanding the LD concept.

In this study, the manufacturing data at hand describe the manufacturing process, the necessary equipment, the sequence of manufacturing processes and the production assets. Production assets are collected in boxes, and the production assets in the production 'line' may be at different levels of completion. In the production environment under review, a great number of different products are manufactured, and each product undergoes several hundred production steps (Osswald et al., 2013). The planned sequence of manufacturing operations for each product are subsumed in a so-called route. Each manufacturing operation can be carried out in several production units, which may be geographically dispersed over the cleanroom environment. The data of each executed manufacturing production process are stored in a relational database. The movement of each asset is tracked by an indoor positioning system based on ultrasound that logs its precise position throughout the entire production process (Dierkes \& Fleisch, 2006). The indoor positioning system stores the data in a separate database/solution.

Spatial data for indoor manufacturing purposes are quite scarce; the spatial dimension has only recently become a focus for manufacturing industries. Hence, manufacturing equipment and sensors in the production line do not report the precise position. In order to overcome this lack of spatial data, we created a spatial dataset, representing the manufacturing environment under review, based on a dataset originating from a computer-aided design system. This spatial dataset (Scholz \& Schabus, 2014; Schabus \& Scholz, 2015) enables the possibility of linking non-spatial attributive data and the spatial dimension, which paves the way for spatial analysis capabilities. For indoor navigation purposes, we derived a network representation - i.e. a graph consisting of edges and nodes.

The general concept of the LD approach in this paper, which combines three datasets, is depicted in Figure 1. First, the IndoorOntology::Production (in blue) serves as a semantic reference for the LD. Second, the manufacturing data (in yellow) are compiled from different data sources. Spatial data (in green) are cross-sectional, and show defined links from the indoor ontology and the manufacturing data. These links ensure that each phenomenon described by LD can be referenced to the spatial dimension.

In detail, spatial data exist for:

- the indoor space, for routing purposes (nodes and edges)

- each production asset's trajectory (as points in a temporal sequence)

- each manufacturing device (and the connection to the indoor space - i.e. nodes)

- restrictions. 
This approach facilitates an integrated spatio-temporal analysis of manufacturing data, as well as an exchange of data and information. Because attributive data are linked to the ontology, each individual dataset is amended with semantic information, which is necessary to link datasets from different systems (e.g. quality assurance vs. manufacturing system).



Figure 1: LD approach for manufacturing data. The indoor navigation ontology is marked in blue, the manufacturing data in yellow, and the spatial data in green. The arrows denote typed links between datasets and classes.

\section{Linked Manufacturing Data - a Detailed View}

This section highlights details of the data and the chosen LD approach, and shows the relations to the indoor ontology and the spatial data at hand. We restrict ourselves to significant examples that show the complexity present in this area of application.

Production assets are the main objects of interest in this manufacturing environment. In the case of this specific semiconductor manufacturing company, relevant datasets for production assets are mostly stored in various systems or in unconnected databases. Figure 2 depicts the LD approach for each production asset. Each asset is connected with typed links to a single route, which describes the sequence of manufacturing operations to be carried out. In addition, each manufacturing operation can be executed by one or more pieces of equipment (see also Figure 4). In the cleanroom, manufacturing devices are geographically dispersed 
over the production hall. Thus, the position of each device and the position of the production asset is of particular interest for finding the nearest machine for the next operation to be carried out. Therefore, the trajectory is linked to each production asset, including the spatio-temporal dimension. This guarantees a temporally ordered sequence of points with coordinates. The trajectory of an asset subsumes tracked asset positions, representing the movement of the asset through the production line. In addition, the manufacturing processes carried out and the corresponding equipment are linked to the production asset to offer the possibility of retrieving historic information for analysis.

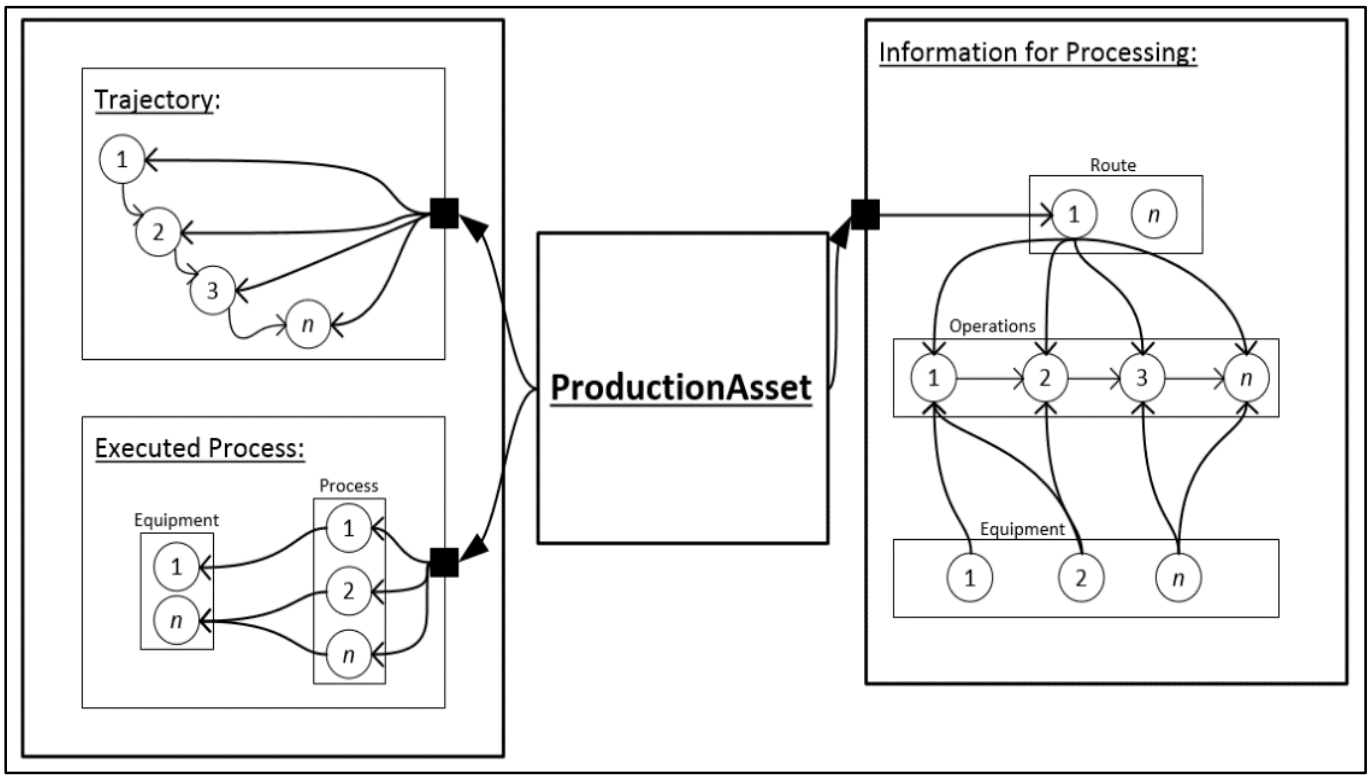

Figure 2: Datasets linked to each individual production asset.

Figure 3 gives a digital representation of a production asset, highlighting the linked information for each production asset. The figure is similar to Figure 2, but shows the typed linkages between the pieces of information, as well as the link to the abstract class 'ProductionAsset' (in blue) - which is a result of the IndoorOntology::Production. An individual production asset is equivalent to an individual in the ontology used. This is similar to an instance of an abstract class in software engineering. In addition, the structure of the attached trajectories is given, with points given in temporal order by the 'nextPosition' link. Executed processes (in red), are also stored in a temporal sequence, but here the system calculates the ordering by looking at the start and end times of each process. 


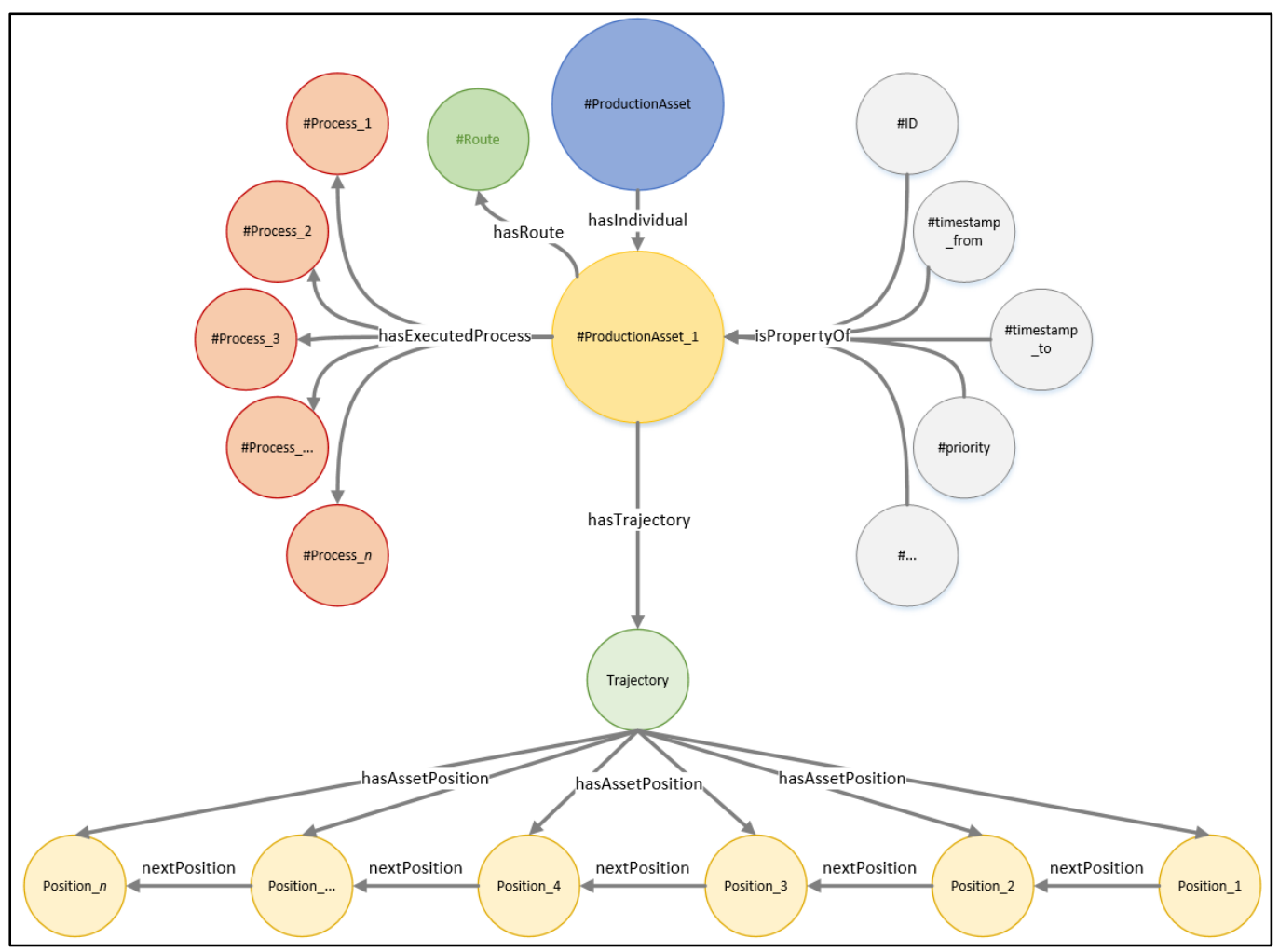

Figure 3: Digital representation of production assets and their linked datasets. Each individual production asset (in yellow) has a unique trajectory (in light green), where the points (in light yellow) are stored in a temporal sequence, denoted with the typed link 'nextPosition'. A unique Route is part of each production asset (in green). Each production asset has properties, which are marked in grey. Executed Processes are marked in light red.

Figure 4 shows a detailed view of the routes. Each production asset has a unique route, representing the manufacturing operations to be carried out. The example of a specific route, named 'Route_X', is linked to three operations/processes via the typed link 'hasOperation'. The temporal sequence of the operations is determined by the typed link 'nextOperation'. The execution of each operation is not restricted to one single manufacturing device, but can be done on several pieces of equipment, shown in yellow circles. 


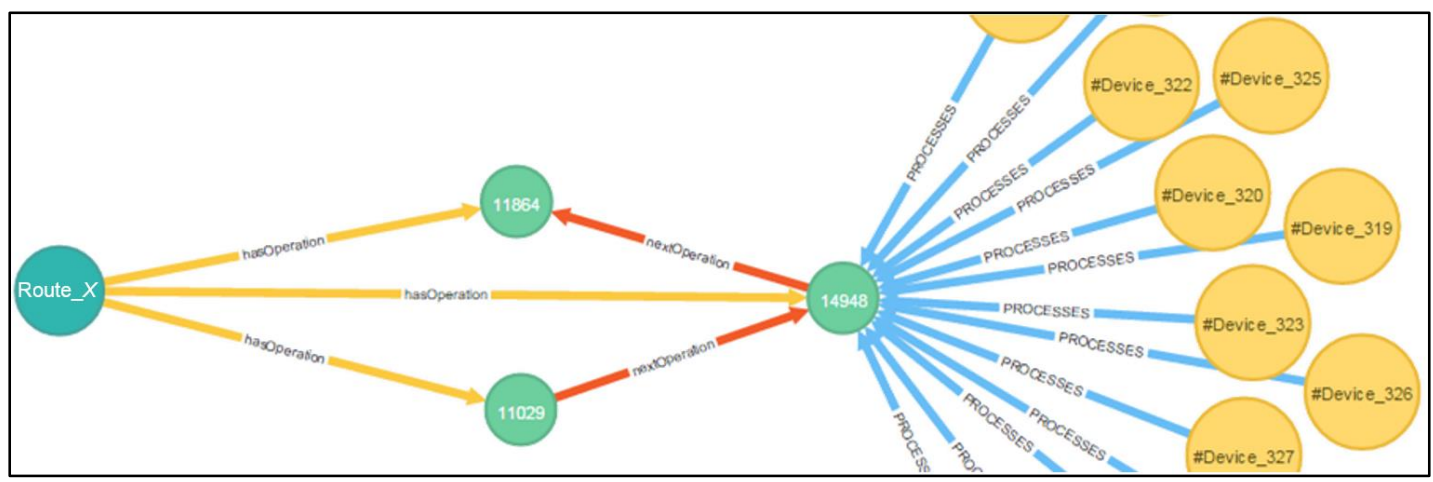

Figure 4: Detailed outline of a production asset and the associated Route. Here the specific Route, 'Route- $X$ ', has a number of attached operations, linked with 'hasOperation'. The operations are in sequence, shown by the typed links 'nextOperation'. Each operation is additionally linked to the devices that are capable of executing the specific task.

\section{Implementation}

In this section, we elaborate on the implementation of a spatial graph database, which is a digital representation of the Universe of Discourse as LD.

A general introduction to graph databases can be found in Robinson et al. (2015). Lampoltshammer and Wiegand (2015) show a successful combination of ontologies and graph databases in the context of GIScience. In this research, we use the graph database Neo4j to implement the LD approach described above. The spatial graph database we developed includes the semantic description of the manufacturing data, the indoor space and the navigation processes. It also incorporates space and time (Cattuto et al., 2013; Pluciennik \& Pluciennik-Psota, 2014). The capability of Neo4j to store and query data with a spatiotemporal dimension leverages the potential for spatio-temporal analysis.

The Sesame framework (Broekstra et al., 2002) is suited for storing and querying RDF data residing in a graph database, in particular a Neo4j database. (This framework has recently been moved to the successor project, RDF4J

(https://projects.eclipse.org/projects/technology.rdf4j).) The framework allows the querying of RDF data using SPARQL, or via RESTful services.

\section{Data Analysis based on Linked Manufacturing Data}

This section explores analysis examples of linked manufacturing data. The examples presented are based on the LD stored in the Neo4j spatial graph database. The data analysis focuses on the one hand on historic information, processing information and network routing capabilities, and on the other hand on the analysis of similarities based on data semantics and the spatial graph database developed during research. 


\section{Analysis of Linked Manufacturing Data}

In order to show the linkage of a route, the associated operations and the possible processing equipment are illustrated in Figure 5. On the left-hand side, the displayed route, "Route_Y", is linked to three operations via the relationship 'hasOperation'. The sequence of operations is shown via the relationship 'nextOperation'. The relationship 'PROCESSES' links operations to possible processing equipment. The right-hand side of the figure illustrates how devices can carry out either a single operation or a variety of operations, and, vice versa, how an operation can be executed by a variety of devices.

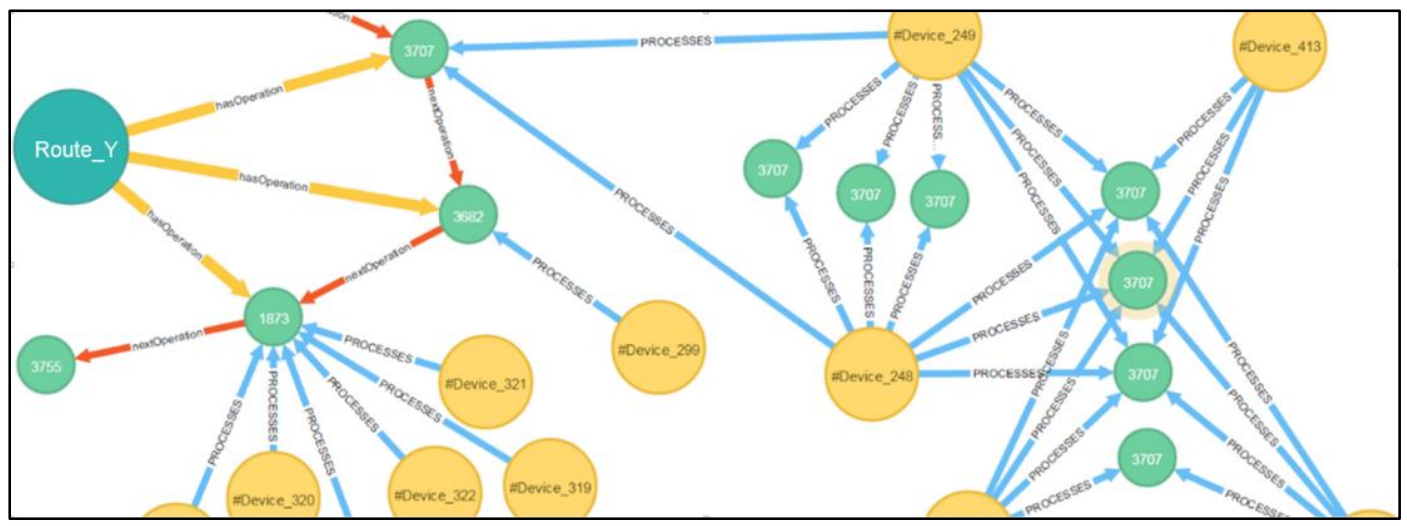

Figure 5: Manufacturing data showing the linkage from a production asset's route, the corresponding sequence of equipment, and possible processing equipment.

Historic information is necessary for quality assurance, monitoring and reporting. Therefore, Figure 6 shows the production asset as the focal point of the visualization. A production asset has several executed processes, and each executed process is linked via the relationship 'hasExecutedProcess'. The stored executed processes include the temporal order, given via timestamps for the processing tasks carried out, to enable successful monitoring. Via the relationship 'ProcessedBy', the spatial graph database stores the link to the device which processed the asset. Figure 6 illustrates how one device was used in several manufacturing steps. 


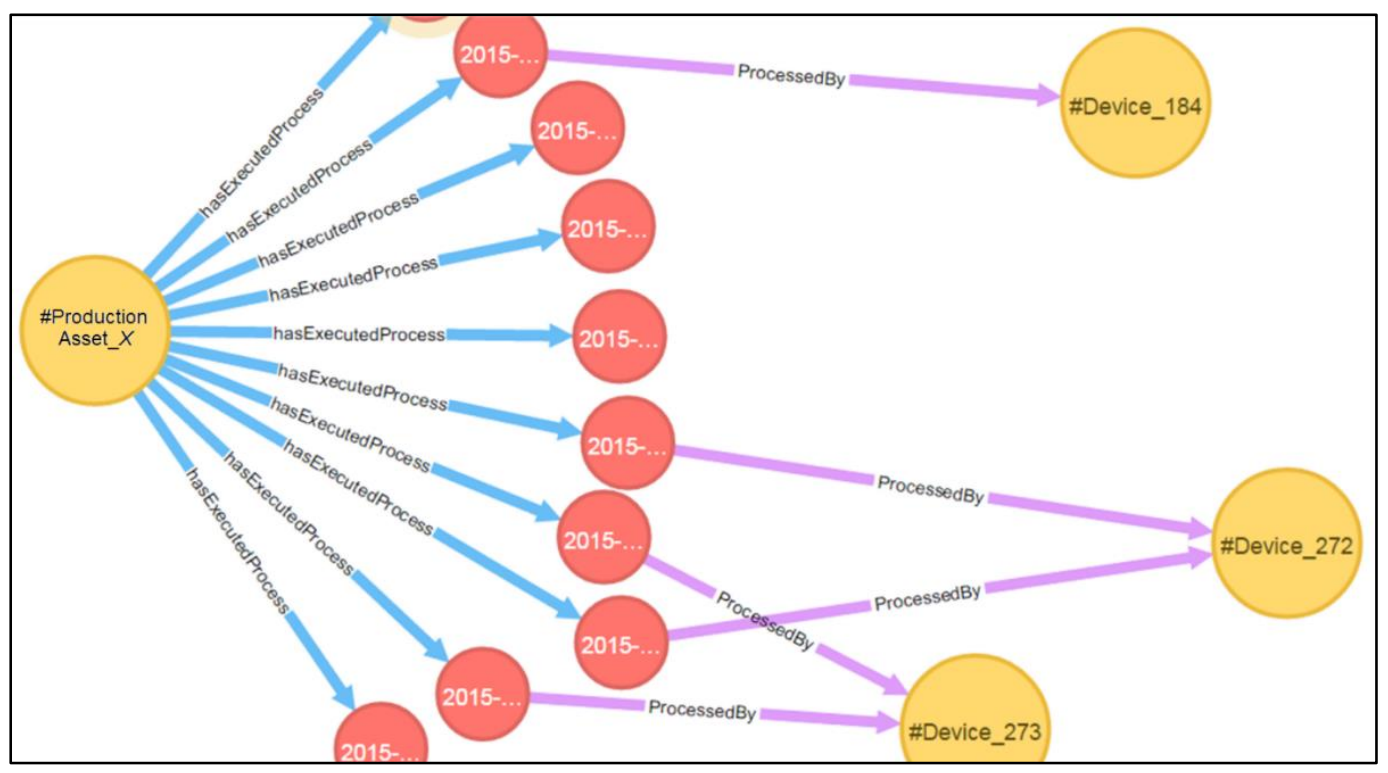

Figure 6: A single production asset 'ProductionAsset_X' and its executed processes, showing that some processes are executed by the same production device.

Network information is important for smart manufacturing purposes and thus especially for the autonomous transportation of production assets within a production line. Figure 7 illustrates the modelling of a routable graph-based network. This network which consists mainly of non-spatial nodes and relationships in the graph database that enable monitoring. For routing purposes, the network is established via the red edges, visualized in Figure 7, and grey nodes (corridor nodes, device nodes) that are semantically annotated. Each of these nodes is linked via the relationship 'hasSpatialNode' to a spatial object in order to enable visualization and analysis. Similarly, the relationship 'linkedTo' binds the incoming/outgoing edges to each node for the spatial view, which is then added via the relationship 'hasSpatialEdge'. 


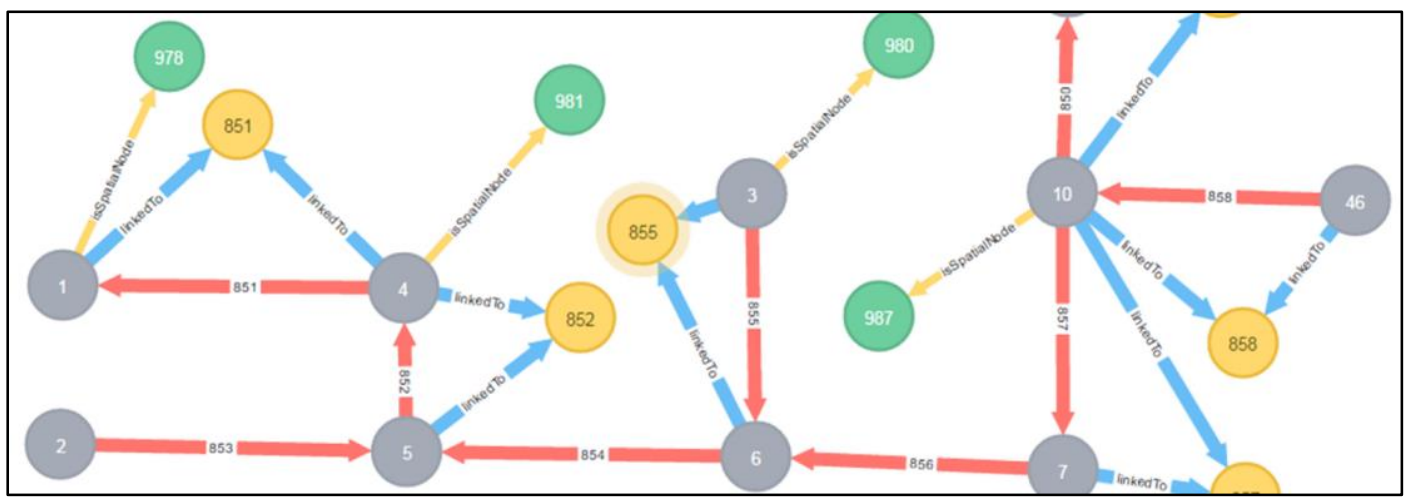

Figure 7: Routable Network in the spatial graph database and a link to spatial information, including the capability to link spatial suitability to and corresponding affordances to a potential route optimization.

\section{Similarity Analysis of Linked Manufacturing Data}

Figure 8 shows an example of a similarity analysis based on semantically annotated linked manufacturing data. This figure focuses on the similarity of the types of production assets. Therefore, the spatial graph database is queried to identify paths between assets based on typed links such as 'from Type' and 'hasSubType'. By assuming that the length of the path or the edge count between assets is affecting the similarity of assets, Asset_1 and Asset_2 (3Edges) are more similar than Asset_1 and Asset_3 (4-Edges). In addition, Asset_1 and Asset_5 are completely different, as there is no possible connection via the defined relationships. This type of similarity analysis can be useful for monitoring quality issues and identifying assets affected after a possible incident. 


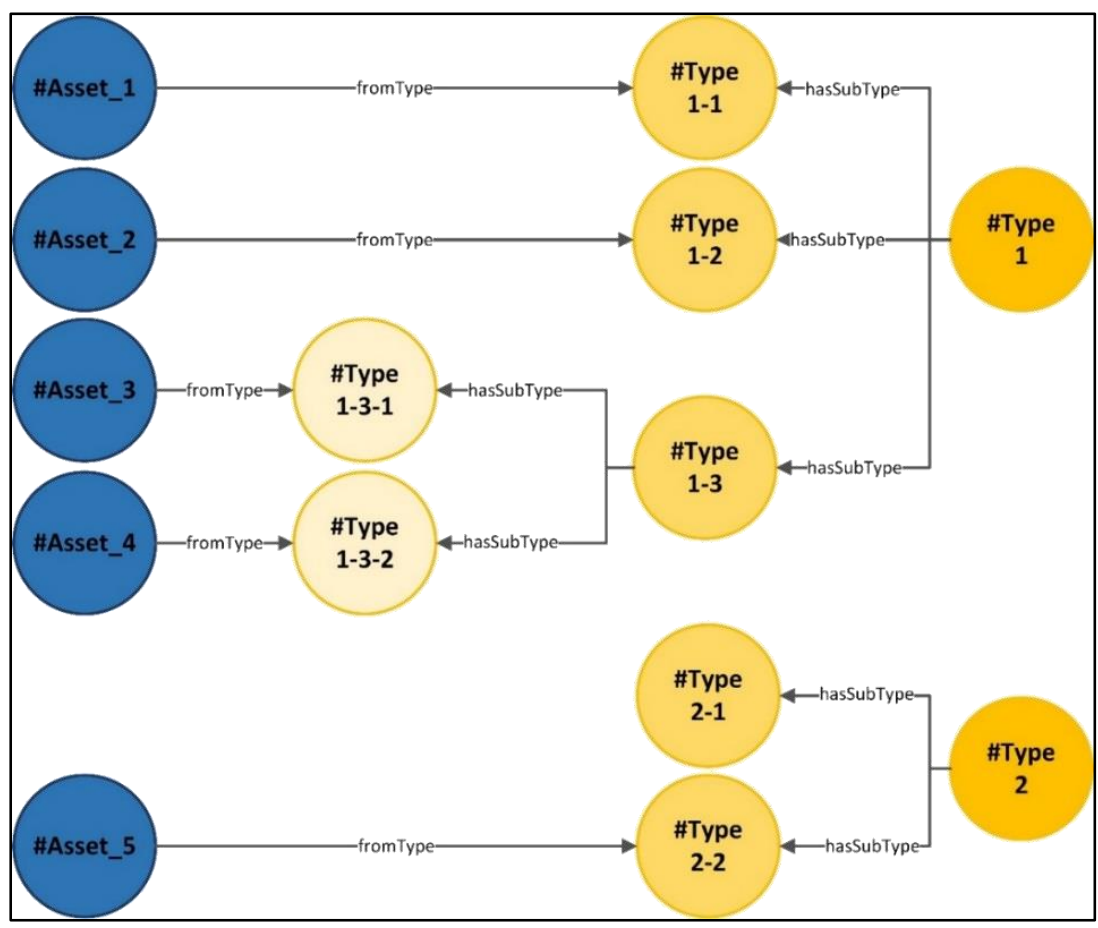

Figure 8: Similarity Example based on the Semantic Annotation of Asset (Sub)-Types.

\section{Conclusion and Discussion}

This paper elaborates on an approach to migrate manufacturing data into a LD approach. This utilizes the expressive power of semantics to annotate manufacturing data. The approach makes use of an ontology that describes the indoor space and the manufacturing process, including real-world objects and processes such as transport, navigation, production assets and devices. The research includes the representation of manufacturing data in a spatial graph database and publishes them to support RESTful services: as 'raw' data in the spatial graph database (queryable via Cypher). Because the data in the graph database are accessible via web-based services (i.e. RESTful services), the data can be reused within an organization. Currently, data discovery and/or data sharing present shortcomings in manufacturing organizations. These issues could be resolved to some degree with the help of semantic interoperability. The analysis capabilities support the evaluation of historical data as well as the autonomous transport of production assets in a smart manufacturing environment.

In answer to the research question 'Does the translation of relational manufacturing data into semantically annotated linked data contribute to new knowledge generation based on data links and data analysis, i.e. identification of similarities?', the authors conclude that a LD approach can contribute to an increase of the spatio-temporal analysis capabilities. The conclusion is justified by the possibility of the LD approach to share data, using a 
standardized interface via web-based services. The LD approach may be beneficial for large manufacturers with vast amounts of data who could utilize semantically tagged data. This enables any user to gain new insights and extract similarities for a given question, based on the explicit semantics of the data. The LD approach can be used to query - and combine datasets from servers which are geographically dispersed. Machines are able to collect data automatically, and perform reasoning tasks with the help of the semantics. In combination with web-based services, this enables seamless data-sharing, overcoming organizational borders in both the syntactic and the semantic dimensions.

\section{References}

Andrienko, G., Andrienko, N., Jankowski, P., Keim, D., Kraak, M.-J., MacEachren, A., \& Wrobel, S. (2007). Geovisual analytics for spatial decision support: Setting the research agenda. International Journal of Geographical Information Science, 21(8), 839-857.

Broekstra, J., Kampman, A., \& Van Harmelen, F. (2002). Sesame: A generic architecture for storing and querying rdf and rdf schema. In The Semantic Web-ISWC 2002 (pp. 54-68). Springer.

Boole, G. (1854). An investigation of the laws of thought: on which are founded the mathematical theories of logic and probabilities. Dover Publications.

Cattuto, C., Quaggiotto, M., Panisson, A., \& Averbuch, A. (2013). Time-varying social networks in a graph database: a Neo4j use case. First International Workshop on Graph Data Management Experiences and Systems, (p. 11).

Compieta, P., Di Martino, S., Bertolotto, M., Ferrucci, F., \& Kechadi, T. (2007). Exploratory spatiotemporal data mining and visualization. Journal of Visual Languages \& Computing, 18(3), 255-279.

Davis, E. (1990). Representations of commonsense knowledge. Morgan Kaufmann.

Davis, J., Edgar, T., Porter, J., Bernaden, J., \& Sarli, M. (2012). Smart manufacturing, manufacturing intelligence and demand-dynamic performance. Computers \& Chemical Engineering, 47, 145-156.

De Mauro, A., Greco, M., \& Michele, G. (2016). A Formal definition of Big Data based on its essential Features. Library Review, 65, 122-135. doi: 10.1108/LR-06-2015-0061.

Dierkes, M., \& Fleisch, E. (2006). LotTrack: RFID-based process control in the semiconductor industry. IEEE Pervasive Computing(1), 47-53.

Goetz, M., \& Zipf, A. (2011). Formal definition of a user-adaptive and length-optimal routing graph for complex indoor environments. Geo-Spatial Information Science, 14(2), 119-128.

Grenon, P., \& Smith, B. (2004). SNAP and SPAN: Towards dynamic spatial ontology. Spatial cognition and computation, 4(1), 69-104.

Gruber, T. R. (1993). A translation approach to portable ontology specification. Knowledge Acquisition, 5(2), 199-220.

Howell, I., \& Batcheler, B. (2005). Building information modeling two years later--huge potential, some success and several limitations. The Laiserin Letter, 22, 4.

Janowicz, K., Scheider, S., \& Adams, B. (2013). A geo-semantics flyby. In Reasoning web. Semantic technologies for intelligent data access (pp. 230-250). Springer.

Klepeis, N. E., Nelson, W. C., Ott, W. R., Robinson, J. P., Tsang, A. M., Switzer, P., \& Engelmann, W. H. (2001). The National Human Activity Pattern Survey (NHAPS): a resource for assessing exposure to environmental pollutants. Journal of exposure analysis and environmental epidemiology, 11(3), 231-252.

Klien, E. (2007). A Rule-Based Strategy for the Semantic Annotation of Geodata. Transactions in GIS, 11(3), 437-452. 
Kuhn, W., Kauppinen, T., \& Janowicz, K. (2014). A Paradigm Shift for Geographic Information Science. A Paradigm Shift for Geographic Information Science. 8728, pp. 173-186. Springer Verlag.

Lampoltshammer, T. J., \& Wiegand, S. (2015). Improving the Computational Performance of Ontology-Based Classification Using Graph Databases. Remote Sensing, 7(7), 9473-9491.

Lutz, M., Sprado, J., Klien, E., Schubert, C., \& Christ, I. (2009). Overcoming semantic heterogeneity in spatial data infrastructures. Computers \& Geosciences, 35(4), 739-752.

Nyström, R. H., Harjunkoski, I., \& Kroll, A. (2006). Production optimization for continuously operated processes with optimal operation and scheduling of multiple units. Computers \& chemical engineering, 30(3), 392-406.

Osswald, S., Weiss, A., \& Tscheligi, M. (2013). Designing wearable devices for the factory: Rapid contextual experience prototyping. Collaboration Technologies and Systems (CTS), 2013 International Conference on, (pp. 517-521).

Pluciennik, T., \& Pluciennik-Psota, E. (2014). Using graph database in spatial data generation. In ManMachine Interactions 3 (pp. 643-650). Springer.

Raubal, M., \& Worboys, M. (1999). A formal model of the process of wayfinding in built environments. In Spatial information theory. Cognitive and computational foundations of geographic information science (pp. 381-399). Springer.

Robinson, I., Webber, J., \& Eifrem, E. (2015). Graph Databases: New Opportunities for Connected Data. O'Reilly Media, Inc.

Schabus, S., \& Scholz, J. (2015). Geographic Information Science and technology as key approach to unveil the potential of Industry 4.0: How location and time can support smart manufacturing. Informatics in Control, Automation and Robotics (ICINCO), 2015 12th International Conference on, 2, pp. 463-470.

Schabus, S., Scholz, J., \& Lampoltshammer, T. J. (2015). A Space in a Space: Connecting Indoor and Outdoor Geography. Proceedings of 18th AGILE Conference on Geographic Information Science 2015. Lisbon.

Scholz, J., \& Schabus, S. (2014). An Indoor Navigation Ontology for Production Assets in a Production Environment. In M. Duckham, E. Pebesma, K. Stewart, \& A. U. Frank (Eds.). Springer International Publishing. doi:10.1007/978-3-319-11593-1_14

Smith, B. (2001). Objects and their environments: from Aristotle to ecological ontology. The life and motion of socio-economic units: GISDATA, 8.

Sowa J. (2014). Building, sharing and merging ontologies. Retrieved from www.jfsowa.com/ontology/ontoshar.htm [last visited: 17-03-2017].

von Landesberger, T., Brodkorb, F., Roskosch, P., Andrienko, N., Andrienko, G., \& Kerren, A. (2016). MobilityGraphs: Visual Analysis of Mass Mobility Dynamics via Spatio-Temporal Graphs and Clustering. Visualization and Computer Graphics, IEEE Transactions on, 22(1), 11-20.

Yang, L., \& Worboys, M. (2015). Generation of navigation graphs for indoor space. International Journal of Geographical Information Science, 29(10), 1737-1756. doi:10.1080/13658816.2015.1041141

Zuehlke, D. (2010). SmartFactory-Towards a factory-of-things. Annual Reviews in Control , 34(1), 129-138. doi:http://dx.doi.org/10.1016/j.arcontrol.2010.02.008 